\title{
Combined pH-impedance monitoring and high-resolution manometry of Japanese patients treated with proton-pump inhibitors for persistent symptoms of non-erosive reflux disease
}

\author{
Hiroshi Yamashita ${ }^{1}$, Kiyoshi Ashida ${ }^{1}$, Takumi Fukuchi ${ }^{1}$, Yoshiaki NAGATani ${ }^{1}$, \\ Hideaki Koga ${ }^{1}$, Kasane Senda ${ }^{1}$, Takaaki Eguchi ${ }^{1}$, Satoshi Ubukata ${ }^{1}$, \\ Shinpei Kawaguchi ${ }^{1}$, Aya Ueda ${ }^{1}$, Toshio Tanaka ${ }^{1}$, Rina Ohashi ${ }^{1}$ and Dai Ito ${ }^{1}$ \\ ${ }^{1}$ Department of Gastroenterology and Hepatology, Saiseikai Nakatsu Hospital, Japan
}

Received August 1, 2012; Accepted December 6, 2012

\begin{abstract}
Background: Data on acid and non-acid reflux patterns and esophageal function in Japanese patients with non-erosive reflux disease (NERD) are limited. The aim of this study was to use combined multichannel intraluminal impedance $\mathrm{pH}$ monitoring (MII-pH) and high-resolution manometry (HRM) to investigate the characteristics of Japanese patients who were treated with a "double-dose" (20 mg) of rabeprazol (a proton-pump inhibitor; PPI) for persistent symptoms of NERD. Methods: Twenty-five patients who complained of typical gastroesophageal reflux disease symptoms, which had occurred more than twice a week despite treatment with rabeprazol, were enrolled in the study. All patients underwent upper endoscopy, esophageal HRM, and 24-h MII-pH monitoring while double-dose PPI therapy was continued. Results: Twelve $(48.0 \%)$ of the patients had a positive symptom index (SI) with 234 recorded symptoms, 127 (54.3\%) of which were related to reflux episodes. Of those with reflux episodes, 29 (22.8\%) were related to acid reflux, while $98(77.2 \%)$ were the result of a weaker acidic reflux. In acid reflux and in mixed (liquid-gas) reflux, the proximal esophageal region was involved to a significantly greater degree $(P<0.002$ and $P=0.005$, respectively) than the distal region. In liquid reflux, there was no difference between the distal and proximal regions. HRM showed that proximal motility parameters were significantly more defective than in those of healthy volunteers. Conclusions: MII-pH monitoring indicated that weakly acidic reflux and mixed refluxate in the proximal esophagus is the major cause of persistent symptoms in patients with NERD who are being treated with PPI. HRM showed that proximal esophageal dysfunction might be a key condition that facilitates reflux.
\end{abstract}

Key words: non-erosive reflux disease (NERD), combined multichannel intraluminal impedance-pH monitoring (MII-pH), high resolution manometry (HRM), weakly acid reflux, mixed reflux

Correspondence to: Dr. Hiroshi Yamashita, Department of Gastroenterology and Hepatology, Saiseikai Nakatsu Hospital, 2-10-39 Shibata Kita-ku, Osaka 530-0012, Japan

Phone: +81663720333 Fax:+81663720339 e-mail: onakaryoko0310@gmail.com

(C)2012 The Japan Society of Smooth Muscle Research

doi: $10.1540 /$ jsmr.48.125 


\section{Introduction}

Gastroesophageal reflux disease (GERD) is one of the most common chronic gastrointestinal diseases in Western countries. In Japan, the prevalence of GERD began to increase at the end of the 1990s (Fujiwara et al., 2009). Non-erosive reflux disease (NERD) and erosive esophagitis (EE) represent the most common phenotypic presentations of GERD. In particular, NERD, which is defined as the presence of the typical symptoms (ie, heartburn and/or regurgitation) of GERD that are caused by intraesophageal reflux, in the absence of esophageal mucosal injury observed via endoscopy, is estimated to affect between $50 \%$ and $70 \%$ of the whole GERD population (Fujiwara et al., 2009; Kahrilas, 1996; Fass, 2007). In several studies, differences in the following physiologic characteristics between NERD and EE patients have been reported: patients with NERD tend to have normal lower esophageal sphincter (LES) pressures, minimal esophageal body motility abnormalities, low esophageal acid exposure profiles, and minimal night-time esophageal exposure to acid (Fass, 2003; Quigley, 1997; Adachi et al., 2003). Patients with NERD were historically believed to have a milder disease than those with EE. This concept is incorrect, given that the impact on the quality of life is similar in patients with GERD, with or without esophagitis (Fass et al., 2007).

Rabeprazole $(20 \mathrm{mg})$ is the standard dose for reflux esophagitis in Western countries, but is considered to be double-dose PPI therapy in Japan. Dean et al. (2004) reported that the symptomatic response rate to standard $10 \mathrm{mg}$ PPI doses at 4 weeks was only $37 \%$ for patients with NERD. In contrast, patients with EE account for only $30-40 \%$ of the GERD population, and their symptom response rate is significantly greater than what has been reported for patients with NERD (the pooled symptomatic response rate to standard $10 \mathrm{mg}$ PPI dose at 4 weeks was 56\%) (Dean et al., 2004). Failure of PPI treatment to resolve reflux-related symptoms has become the most common presentation of NERD in gastrointestinal practice. The identification of a solution for the persistent symptoms of patients with NERD who are taking PPI is desired.

Recently, the addition of impedance channels to conventional $\mathrm{pH}$ catheters has offered the ability to detect and monitor liquid and air movement within the esophagus and to distinguish between acid and nonacid reflux (Brednoord et al., 2005; Sifrim et al., 2004). In addition, it has been shown that combined multichannel intraluminal impedance $\mathrm{pH}$ monitoring (MII-pH) is more accurate than $\mathrm{pH}$ alone for the detection of both acidic and weakly acidic refluxes, and it is a sensitive method for the detection of the proximal extent of the reflux (Savarino et al., 2008). High-resolution manometry (HRM) is a more accurate method than conventional manometry for evaluating esophageal motility (Pandolfino et al., 2009). In Western countries, the evaluation of NERD is usually determined by using MII-pH and HRM. There are, however, limited data on acid and non-acid reflux patterns and the esophageal function of Japanese patients with NERD (Iwakiri et al., 2009). The aim of this study was to use MII-pH and HRM to investigate the characteristics of Japanese patients with persistent symptoms of NERD who were treated with double doses of PPI. 


\section{Methods}

From July 2010 to July 2011, 25 patients who complained of typical GERD symptoms, such as heartburn and/or regurgitation, that occurred more than twice per week despite treatment with rabeprazole (10 mg twice daily), took part in the study. All patients underwent upper endoscopy, esophageal HRM, and 24-h MII-pH monitoring. Patients were diagnosed with NERD when they had troublesome reflux-related symptoms in the absence of either esophageal mucosal erosion or breaks observed using conventional endoscopy and with no primary esophageal motility disorders. Exclusion criteria were any previous esophageal or gastric surgery.

This observational study was conducted in Saiseikai Nakatsu Hospital from July 2010 to July 2011. The protocol and patients' informed consent forms were reviewed and approved by the ethical committee at Saiseikai Nakatsu Hospital before the study. The study was conducted in accordance with the Declaration of Helsinki, the consolidated Good Clinical Practice guidelines, and the applicable regulatory requirements.

\section{Intraluminal $\mathrm{pH}$ and impedance recording}

The study was conducted after at least $6 \mathrm{~h}$ of fasting. A MII-pH probe was placed in reference to the manometrically located proximal border of the LES using HRM. The configuration of the catheter allowed for the monitoring of changes in the intraluminal impedance at 3, 5, 7, 9, 15, and $17 \mathrm{~cm}$ above the proximal margin of the LES. In addition, $\mathrm{pH}$ was monitored $5 \mathrm{~cm}$ above and $10 \mathrm{~cm}$ below the proximal margin of the LES.

Patients were encouraged to maintain normal activity and sleep schedules at regular times. All patients took the same meals (2,400 kcal/day) during an MII-pH monitoring procedure at the hospital. Data from the impedance channels and the $\mathrm{pH}$ electrodes were sampled at a frequency of $50 \mathrm{~Hz}$ and stored on a portable data recorder (Sleuth; Sandhill Scientific, Highlands Ranch, CO, USA). At the end of the 24-h recording period, the data were transferred and analyzed manually using dedicated software (Bioview Analysis; Sandhill Scientific).

\section{Data analysis of intraluminal $\mathrm{pH}$ and impedance recording}

Meal periods were marked on the recording and excluded from the analysis. Liquid-only reflux episodes were identified as retrograde $50 \%$ declines in impedance from baseline in at least 2 sites. Gas-only reflux episodes (simultaneous increase in impedance over 3,000 ohms in any 2 consecutive sites with 1 site having an absolute value over 7,000 ohms), were not included in the analysis. Mixed liquid-gas reflux episodes were defined as gas reflux occurring immediately before or during a liquid reflux. Impedance-detected reflux episodes were classified as acidic if the $\mathrm{pH}$ sensor recorded a decline in $\mathrm{pH}$ to below 4, weakly acidic if the $\mathrm{pH}$ remained above 4 and below 7, or weakly alkaline if the $\mathrm{pH}$ was above 7 when the bolus was present in the esophagus (Sifrim et al., 200413).

Combined MII-pH data allowed for the separation of symptoms into those associated with acid reflux, weakly acidic reflux, weakly alkaline reflux, or not associated with reflux. A symptom was considered to be associated with reflux if a reflux episode was detected $5 \mathrm{~min}$ before the occurrence of the symptom. The primary end point for the study was the SI (Symptom Index). The SI was defined as the number of symptoms associated with reflux divided by the total number of symptoms. SI was 
considered positive if it was detected to be more than $50 \%$.

\section{Esophageal manometry}

A HRM assembly with 36 solid-state sensors that were spaced at $1 \mathrm{~cm}$ intervals (ManoScan360, Sierra Scientific Instruments, LLC, Los Angeles, CA, USA) was used on all patients before MII-pH monitoring. Each sensor was circumferentially sensitive and zeroed to gastric pressure. The HRM assembly was passed transnasally and positioned in order to record from the hypopharynx to the stomach with about 5 intragastric sensors. The manometric protocol included a 5 -min period to assess basal sphincter pressure and ten swallows of 5-ml water.

Manometric data were analyzed using ManoView (Sierra Scientific Instruments LLC) analysis software. The LES pressures were measured with the electronic sleeve function of the ManoView system. We measured distal and proximal esophageal pressures as an average of the 2 adjacent pressure sensors that were 3 to $10 \mathrm{~cm}$ above LES and 14 to $18 \mathrm{~cm}$ above the LES. In addition, we analyzed the proportions of distal and proximal successful primary peristaltic episodes. The manometric data of positive SI patients were compared to 8 asymptomatic healthy volunteers (HVs).

\section{Statistical analysis}

The statistical analysis was performed using the pooled data, and comparisons of proportions were calculated using the $\chi^{2}$ test. Comparisons of the manometry data between NERD patients and HVs were performed using the Mann-Whitney test. A $P$ value of less than 0.05 was considered to be statistically significant.

\section{Results}

\section{Demographic data}

Twelve (48.0\%; nine men; mean age, 65.5 years; range, 32-86) of the total 25 patients had a positive SI, and they reported 234 symptoms during the study (mean, 19.5; range, 1-44). Five (41.7\%) of the 12 positive-SI patients had a higher SI for acid reflux than for weakly acidic reflux. Seven (58.3\%) had a higher SI for weakly acidic reflux than for acid reflux (Table 1). Only 1 of the positive-SI patients had a hiatal hernia, which was determined by HRM when the LES-crural diaphragm separation was over $2 \mathrm{~cm}$ at inspiration. Abnormal acid exposure ( $\mathrm{pH} 4$ holding time $>4 \%$ ) was shown in only 2 patients.

\section{Reflux Episodes during PPI Therapy}

In the 12 patients with positive SI, 565 liquid only and mixed reflux episodes were recorded. Among these, 153 (27.1\%) were acid reflux, 402 (71.2\%) were weakly acidic reflux and 10 (1.7\%) were weakly alkaline reflux (Fig. 1).

\section{Reflux-Related Symptoms during PPI therapy}

A total of 234 symptoms was recorded by the 12 patients with positive SIs, 127 (54.3\%) of which were related to reflux episodes. Twenty-nine (22.8\%) of the 127 symptomatic reflux episodes were related to acid reflux, and $98(77.2 \%)$ were related to weakly acidic reflux. There was no weakly alka- 
Table 1. Demographic characteristics (age and gender) and individual results of the symptom index (SI) of all symptoms, acid reflux, weakly acidic reflux, and weakly alkaline reflux of the 12 patients who had a positive SI

\begin{tabular}{cccccccc}
\hline & & & & \multicolumn{3}{c}{ Symptom index } \\
\cline { 5 - 7 } Case & Age & Gender & $\begin{array}{c}\text { Number of } \\
\text { symptomatic episodes }\end{array}$ & $\begin{array}{c}\text { All symptoms } \\
(\%)\end{array}$ & $\begin{array}{c}\text { Acid } \\
(\%)\end{array}$ & $\begin{array}{c}\text { Weakly acidic } \\
(\%)\end{array}$ & $\begin{array}{c}\text { Alkaline } \\
(\%)\end{array}$ \\
\hline 1 & 72 & male & 31 & 67.7 & 3.2 & 64.5 & 0 \\
2 & 77 & male & 40 & 50 & 15 & 35 & 0 \\
3 & 55 & male & 1 & 100 & 100 & 0 & 0 \\
4 & 86 & male & 2 & 50 & 50 & 0 & 0 \\
5 & 73 & male & 44 & 56.8 & 0 & 56.8 & 0 \\
6 & 62 & male & 30 & 66.7 & 60 & 6.7 & 0 \\
7 & 73 & female & 19 & 52.3 & 15.8 & 36.8 & 0 \\
8 & 70 & female & 1 & 100 & 100 & 0 & 0 \\
9 & 72 & male & 8 & 50 & 0 & 50 & 0 \\
10 & 60 & male & 5 & 80 & 20 & 60 & 0 \\
11 & 54 & female & 44 & 50 & 40 & 10 & 0 \\
12 & 32 & male & 9 & 67 & 0 & 67 & 0 \\
\hline
\end{tabular}

Twelve (48.0\%) of the total 25 patients had a positive SI and reported 234 symptoms. Five (41.7\%) of the 12 positive SI patients had a higher SI for acidic reflux than for weakly acidic reflux. Seven (58.3\%) had a higher SI for weakly acidic reflux than for acidic reflux.

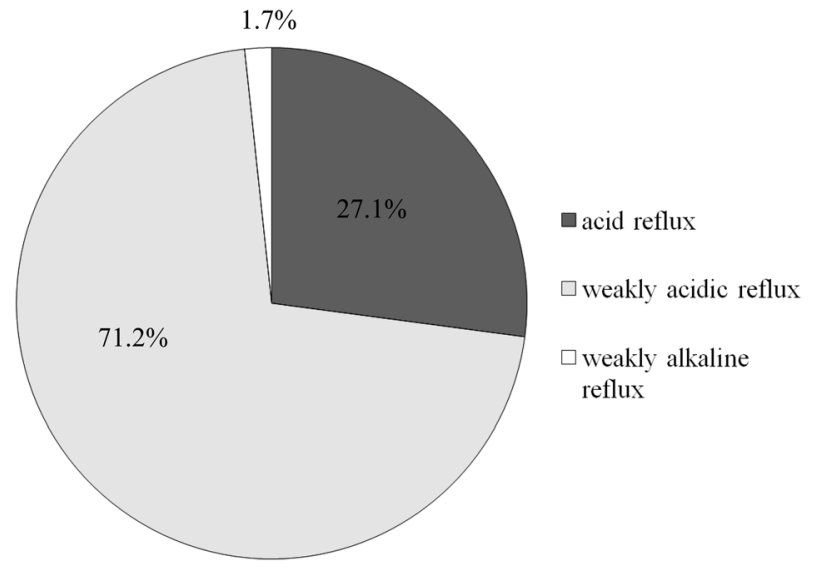

Fig. 1. Proportions of acidity or alkalinity of the symptomatic refluxate in patients with non-erosive reflux disease (NERD) with a positive symptom index (SI) who were treated with doubledose proton-pomp (PPI) therapy.

line reflux associated with reflux symptoms. Therefore, the majority (77.5\%) of reflux episodes during double-dose PPI therapy were asymptomatic, and the majority (77.2\%) of symptomatic reflux episodes were associated with weakly acidic reflux.

\section{Characteristics of symptomatic and asymptomatic reflux}

In symptomatic reflux, $32.3 \%$ were liquid and $67.7 \%$ were mixed (liquid-gas) reflux. In contrast, in asymptomatic reflux, $57.5 \%$ were liquid and $42.5 \%$ were mixed reflux (Fig. 2). The proportion of mixed reflux in symptomatic reflux was greater compared to that in asymptomatic reflux $(P<0.001)$. 


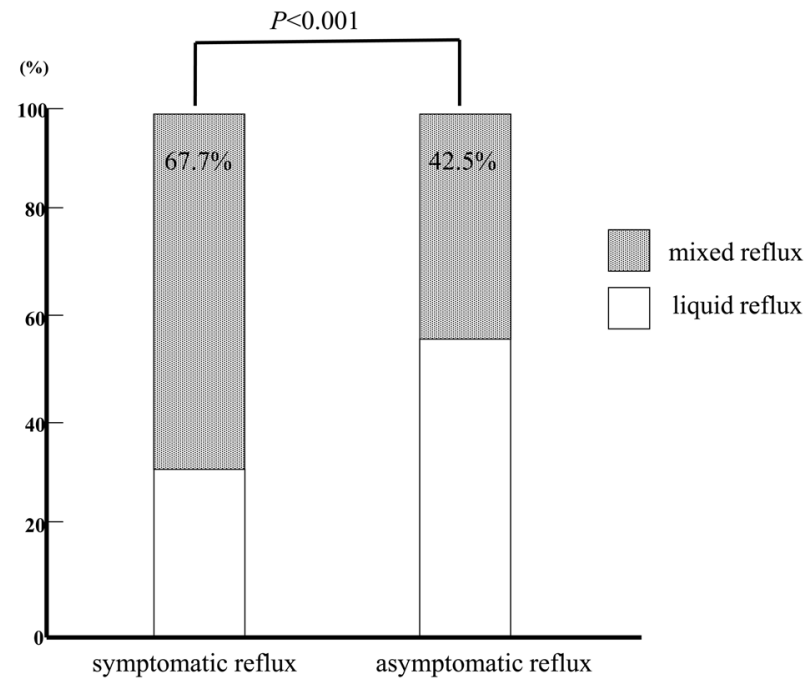

Fig. 2. Proportions of the compositions of symptomatic and asymptomatic reflux in patients with NERD with a positive SI who were being treated with double-dose PPI therapy. The proportion of mixed reflux in symptomatic reflux was greater compared to that of in asymptomatic reflux $(P<0.001)$.

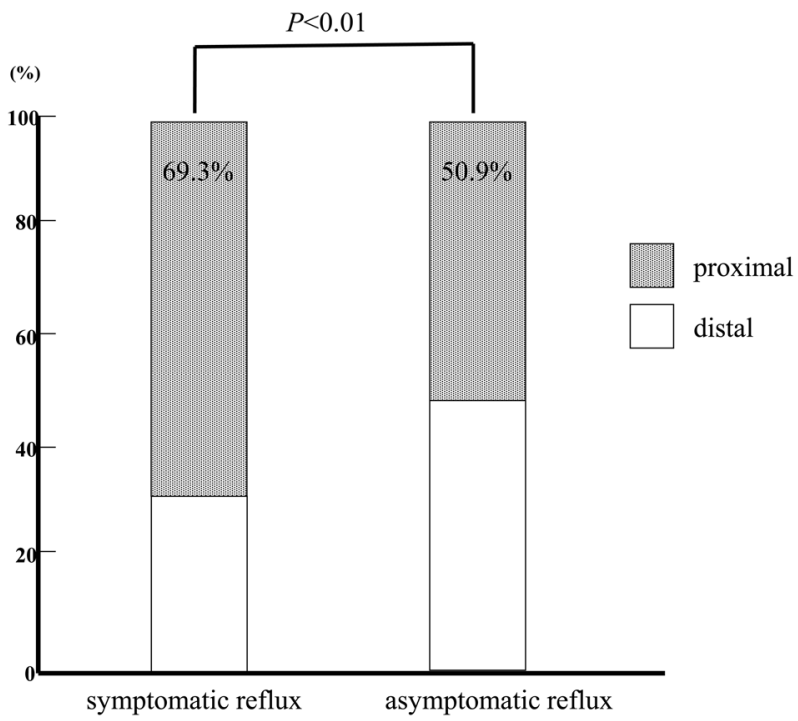

Fig. 3. Proportions of proximal amd distal reflux extents in symptomatic and asymptomatic reflux episodes. Proximal reflux episodes were defined as those, where the refluxate reached 15 or $17 \mathrm{~cm}$ above the lower esophageal sphincter (LES). Proximal reflux episodes in symptomatic reflux were significantly greater $(P<0.01)$ compared to that of asymptomatic reflux.

The proportion of symptomatic reflux episodes at distal sites $(3,5,7$, and $9 \mathrm{~cm}$ above the LES) and at proximal sites (15 and $17 \mathrm{~cm}$ above LES) were $30.3 \%$ and $69.3 \%$, respectively, while they were $49.1 \%$ and $50.9 \%$, respectively, in asymptomatic reflux (Fig. 3). Proximal reflux episodes in symptomatic reflux were therefore significantly greater $(P<0.01)$ compared to that of asymptomatic reflux. 
Table 2. Proportions of symptomatic reflux according to the acidity and composition at each proximal and distal esophageal site

\begin{tabular}{llccc}
\hline & & \multicolumn{3}{c}{ Proportions of symptomatic reflux (\%) } \\
\cline { 3 - 5 } & & \multicolumn{1}{c}{ distal } & proximal & $P$ value (proximal $v$ s. distal) \\
\hline \multirow{2}{*}{ acidity } & acid & $2.5 \pm 1.9$ & $19.7 \pm 8.4$ & $P<0.002$ \\
& weakly acidic & $37.0 \pm 8.2$ & $40.8 \pm 7.6$ & N.S \\
\multirow{2}{*}{ composition } & liquid & $14.4 \pm 7.6$ & $24.4 \pm 8.1$ & N.S \\
& mixed & $15.4 \pm 3.7$ & $45.8 \pm 8.1$ & $P=0.005$ \\
\hline
\end{tabular}

Data are expressed as mean $\pm \mathrm{SE}$. Analysis was performed using the Fisher's exact test. $P$ values less than 0.05 were considered statistically significant. N.S, not significant.

Table 3. High-resolution manometry results the patients with nonerosive reflux disease (NERD) compared to healthy volunteers (HVs)

\begin{tabular}{lccc}
\hline & NERD $(\mathrm{n}=12)$ & HV $(\mathrm{n}=8)$ & $P$ value \\
\hline Proximal primary peristalsis $(\mathrm{mmHg})$ & $34.5(14.7-61.9)$ & $47.4(37.5-73.3)$ & $<0.05$ \\
Proximal successful peristalsis (\%) & $63.8(10-100)$ & $95.2(80-100)$ & $<0.02$ \\
Distal primary peristalsis (mmHg) & $78.6(37.5-128.7)$ & $83.5(63.4-141.3)$ & N.S \\
Distal successful peristalsis (\%) & $90.0(60-100)$ & $94.5(80-100)$ & N.S \\
LES pressure (mmHg) & $10.5(9.3-18.3)$ & $12.0(10.3-21.2)$ & N.S \\
Residual LES pressure (mmHg) & $2.8(2.1-8.8)$ & $3.2(2.9-10.5)$ & N.S \\
\hline
\end{tabular}

Data are expressed as median (interquartile range). Analysis was performed using the Mann-Whitney test. $P$ values less than 0.05 were considered statistically significant. LES, lower esophageal sphincter; N.S, not significant.

The proportions of symptomatic reflux according to the acidity and composition of the refluxate are shown in Table 2 . The majority of symptomatic reflux episodes were weakly acidic, and there was no difference in the proportions of weakly acidic symptomatic reflux episodes between distal and proximal sites. However, for acid reflux, the proximal extent was involved significantly more $(P<0.002)$ than the distal extent. For the proportions of symptomatic reflux according to the composition, there was no difference in the proportion of symptomatic liquid reflux between distal and proximal sites. For mixed reflux, the proximal extent was significantly more involved $(P=0.005)$ than the distal extent.

\section{Esophageal manometry}

In NERD patients, the proximal primary peristaltic pressure and the proximal successful peristaltic ratio were significantly lower $(P<0.05$ and $P<0.02$, respectively) than in HVs. However, there were no differences in the in distal primary peristaltic pressure and the distal successful peristaltic ratio between NERD patients and HVs. In addition, there were no differences in the mean LES pressure and the residual LES pressure between NERD patients and HVs (Table 3).

\section{Discussion}

In this study, we investigated the reflux patterns of Japanese patients with NERD while they were being treated with double-dose PPI therapy using 24-h combined MII-pH monitoring. Combined MII- 
$\mathrm{pH}$ monitoring in the 12 patients with a positive SI showed that reflux-related symptoms occurred in $22.5 \%$ of reflux episodes and $54.3 \%$ of reflux symptoms were related to reflux episodes (acid/weakly acidic reflux). In addition, $22.8 \%$ were related to acid reflux, and $77.2 \%$ were related to weakly acidic reflux. Therefore, weakly acidic reflux is the major mechanism that underlies persistent symptoms in patients with NERD who are being treated with PPI therapy.

In patients with GERD who were not treated with PPI, $63 \%$ of reflux episodes were acidic and $72 \%$ of reflux-related symptom episodes were associated with acidic reflux (Hershcovici et al., 2012). PPI therapy has been shown to convert predominantly acidic reflux to weakly acidic reflux in studies that were performed on HVs and GERD patients (Tamhankar et al., 2004; Vela et al., 2001), and this appears to occur without a reduction in the total number of reflux episodes. Weakly acidic reflux is undetectable by conventional $\mathrm{pH}$ monitoring, MII-pH monitoring must be done to evaluate reflux episodes in patients being treated with PPI therapy. The aim of this study was to investigate the characteristics of patients with NERD with persistent symptoms who were treated with double-dose PPI therapy. Therefore, we investigated the use of MII-pH monitoring and HRM in patients with NERD on PPI therapy.

Studies have shown that the proximal extent of the refluxate is an important factor in the perception of reflux (Zerbib et al., 2008; Emerenziani et al., 2009). In addition, Iwakiri et al. reported that reflux episodes in which the refluxate reaches the proximal esophagus were significantly more likely to be perceived as a symptom than reflux episodes that are confined to the distal esophagus (Iwakiri et al., 2010). Indeed, the present study showed that the proportion of proximal reflux in symptomatic reflux episodes was significantly higher than that in asymptomatic reflux episodes. With regard to the characteristic of symptomatic reflux episodes, the proportion of weakly acidic reflux was higher than that of acid reflux (77.2\% vs. $22.8 \%$ ) and the proportion of mixed reflux was higher than that of liquid reflux $(67.7 \%$ vs. $32.3 \%)$. Thus, weakly acidic reflux is the major mechanism for NERD, but there was no difference in the proportions of symptomatic reflux between distal and proximal sites in weakly acidic reflux. However, in acid reflux, the proximal extent was significantly more involved $(P<0.002)$ than the distal extent. We showed that the presence of acid in the refluxate tends to occur in the proximal esophagus. For the proportions of symptomatic reflux according to the composition, there was no difference in the proportions of symptomatic reflux between distal and proximal sites for liquid reflux. For mixed reflux, the involvement of the proximal extent was significantly greater $(P=0.005)$ than that of the distal extent. Therefore, these results suggest that the refluxate containing gas tended to reach the proximal esophagus.

Esophageal hypersensitivity is considered to be one of the factors of symptomatic reflux episodes in patients with NERD. In a recent study, stimulus response to acid and saline infusion was quantified and it was demonstrated that NERD patients in particular exhibit esophageal hypersensitivity not only to acid, but also to saline perfusion, suggesting that hyperalgaesia to acid and other factors may play a role in symptom generation in these patients (Nagahara et al., 2006). These findings support the concept of a higher sensitivity of NERD patients to acid and weakly acidic reflux. Ribolsi et al. (2008) reported that a brief duration of acid exposure sensitizes the esophagus to the perception of weakly acidic reflux in patients with NERD.

The present study showed that the proportion of mixed reflux was higher than the proportion of liquid reflux in symptomatic reflux episodes. Emerenziani et al. (2008) demonstrated that the presence of gas in the refluxate significantly enhances the probability of reflux perception in NERD patients who 
also appear to be sensitive to perceiving weakly acidic reflux. It is likely that the presence of gas in addition to liquid increases the volume of the refluxate and, thus, the degree of esophageal distention. Several types of mechano- and chemoreceptors have been identified as being overexpressed and active in NERD, including the transient receptor potential vanilloid 1 (TRPV1) and the protease-activated receptor 2 (PAR2) (Bhat et al., 2006). Using a protocol of stepwise isobaric distention, Karamanolis et al. (2008) demonstrated that the striated muscle esophagus is more compliant and sensitive compared to the distal smooth muscle esophagus. Distention-induced esophageal sensations are caused by esophageal mechanoreceptor excitation, and esophageal compliance might influence the proximal extent and accumulation of the refluxate. We suspect that differences in the distribution of these receptors and compliance of the esophagus might exist between the proximal and distal esophagus. Therefore, weakly acidic and mixed refluxate in the proximal esophagus might be the major mechanism causing the symptoms in patients with NERD who are being treated with PPI therapy.

The muscular wall of the upper one-third of the esophagus is composed of striated muscle, while that of the lower two-thirds of the esophagus is composed of smooth muscle. The transition between them is identified as the transition zone where the proximal contraction wave originating in the striated esophagus terminates and below which a distal contraction wave simultaneously forms and propagates into the distal smooth muscle esophagus (Ghosh et al., 2006). Ghosh et al. (2008) reported that weak mid esophageal contractions at the proximal smooth-muscle segment appear to be the cause of impaired clearance function. Martinek et al. (2008) reported no difference between NERD and mild to moderate erosive esophagitis in terms of motor abnormalities. However, this study was performed using conventional monometry. In the present study, HRM demonstrated that, in NERD patients, the primary peristaltic pressure and a successful peristaltic ratio at the proximal esophagus was lower than those of HVs. On the other hand, there was no difference between patients with NERD and HVs in primary distal peristaltic pressure, successful distal peristaltic ratio, or LES function. Therefore, proximal esophageal dysfunction might allow for the entry of refluxate into the proximal esophagus, and delayed clearance of the refluxate might stimulate several types of mechano- and chemoreceptors.

In conclusion, MII-pH monitoring showed that the movement of weakly acidic or mixed refluxate into the proximal esophagus is the major mechanism causing the persistent symptoms in patients with NERD who are being treated with PPI therapy. HRM revealed disordered motility of the proximal esophagus. Proximal esophageal dysfunction might be the key condition that allows reflux into the proximal esophagus.

Conflict of interest: None.

\section{References}

Adachi, K., Hashimoto, T., Hamamoto, N., Hirakawa, K., Niigaki, M., Miyake, T., Tanimura, H., Ono, M., Kaji, T., Suetsugu, H., Yagi, J., Kamazawa, Y., Mihara, T., Katsube, T., Fujishiro, H., Shizuku, T., Hattori, S., Yamamoto, S. and Kinoshita, Y. (2003). Symtom relief in patients with reflux esophagitis:comparative study of omeprazole,lansoprazole and rabeprazole. J. Gastroenterol. Hepato. 18: $1392-1398$.

Bhat YM. and Bielefeldt K. (2006). Capsaicin receptor (TRPV1) and non-erosive reflux disease. Eur J. Gastro- 
enterol. Hepatol. 18: 263-270.

Brednoord, A.J., Weusten, B.J. and Sifrim, D. (2005). Reproducibility of multichannel intraluminal electrical impedance monitoring of gastroesophageal reflux. Am. J. Gastroenterol. 100: 265-269.

Dean BB., Gano AD Jr., Knight K., Ofman JJ. and Fass R. (2004). Effective of proton pump inhibitors in nonerosive reflux disease. Clin. Gastroenterol. Hepatol. 2: 656-664.

Emerenziani, S., Ribolsi, M., Sifrim, D., Bkondeau, K. and Cicala M. (2009). Regional oesophageal sensitivity to acid and weakly acidic reflux in patients with non-erosive reflux disease. Neurogastroenterol. Motil. 21, 253-258.

Emerenziani, S., Sifrim, D., Habib, F.I., Ribolsi, M, Guarino, M.P.L., Rizzi, M., Caviglia, R., Petitti, T. and Cicala, M. (2008). Presence of gas in the refluxate enhances reflux perception in non-erosive patients with physiological acid exposure of the oesophagus. Gut. 57: 443-447.

Fass, R. (2007). Erosive esophagitis and nonerosive reflux disease (NERD): comparison of epidemiologic, physiologic, and therapeutic characteristics. J. Clin. Gastroenterol. 41: 131-137.

Fass R. (2007). Symptom assessment tools for gastroesophageal reflux disease (GERD) treatment. J. Clin. Gastroenterol. 41: 437-444.

Fass, R. (2003). Epidemiology and pathophysiology of symptomtic gastroesophageal reflux disease. Am. J. Gastroenterol. 98: S2-S7.

Fujiwara, Y. and Arakawa, T. (2009). Epidemiology and clinical characteristic of GERD in the Japanese population. J. Gastroenterol. 44: 518-534.

Ghosh, S.K., Janiak, P., Schwizer, W., Hebbard, G.S. and Brasseur, J.G. (2006). Physiology of the esophageal pressure transition zone: separate contraction waves above and below. Am. J. Physiol. Gastrointest. Liver Physiol. 290: G568-G576.

Ghosh, S.K., Janiak, P., Fox, M., Schwizer, W., Hebbard, G.S. and Brasseur, J.G. (2008). Physiology of the esophageal transition zone in the presence of chronic bolus retention: studies using concurrent high resolution manometry and digital fluoroscopy. Neurogastroenterol. Mot. 20: 750-759.

Hershcovici, T. and Fass, R. (2012). Step-by-step management of refractory gastroesophageal reflux disease. Dis. Esophagus. 2: 1-10.

Iwakiri, K., Kawami, N., Sano, H., Tanaka, Y., Umezawa, M., Kotoyori, M., Hoshihara, Y. and Sakamoto. C. (2009). Acid and non-acid reflux in Japanese patients with non-erosive reflux disease with persistent reflux symtoms, despite taking a double-dose of proton pump inhibitor:a study using combined $\mathrm{pH}$ impedance monitoring. J. Gastroenterol. 44: 708-712.

Iwakiri, K., Sano, H., Tanaka, M., Kawami, N., Umezawa, M., Futagami, S., Hoshihara, Y., Nomura, T., Miyashita, M. and Sakamoto C. (2010). Charactristics of symptomatic reflux episodes in patients with non-erosive reflux disease who have a positive symptom index on proton pump inhibitor therapy. Digestion. 82: 156-161.

Kahrilas, P.J. (1996). Gastroesophageal reflux disease. JAMA. 276: 983-988.

Karamanolis G., Stevens W., Vos R., Tack J., Clave P. and Sifrim D. (2008). Oesophageal tone and sensation in the transition zone between proximal striated and distal smooth muscle oesophagus. Neurogastroenterol. Motil. 20: 291-297.

Martinek J., Benes M., Hucl T., Drastich P., Stirand P. and Spicak J. (2008). Non-erosive gastroesophageal reflux diseases: no difference with regard to reflux pattern and motility abnormalities. Scand. J. Gastroenterol. 43: 794-800.

Nagahara, A., Miwa, H., Minoo, T., Hojo, M., Kawabe, M., Osada, T., Kurosawa, A., Asaoka, D., Terai, T., Ohkusa, T. and Sato, N. (2006). Increased esophageal sensitivity to acid and saline in patients with nonerosive gastro-esophageal reflux disease. J. Clin. Gastroenterol. 40: 891-895.

Pandolfino J.E., Fox M.R., Brednoord A.J. and Kahrilas P.J. (2009). High-resolution manometry in clinical practice: utilizing pressure topography to classify oesophageal motility abnormalities. Neurogastroenterol. Motil. 21: 796-806.

Quigley, E.M. (1997). Gastroesophageal reflux disease:spectrum or continuum? QJM. 90: 75-78. 
Ribolsi, M., Emerenziani, S., Caviglia, R., Guarino, M. and Cicala, M. (2008). Short duration of acid exposure sensitize the esophagus to perception of weakly acidic and mixed reflux in non-erosive reflux disease (NERD) patients. Gastroenterology. 134: A593.

Savarino, E., Zentilin, P., Tutuian, R., Pohl, D., Casa, D., Frazzoni, M., Cestari, R. and Savarino, V. (2008). The role of non-acid reflux in NERD-lessons learned from impedance-pH monitoring in 150 patients off therapy. Am. J. Gastroent. 103: 2685-2693.

Sifrim, D., Castell, D.O., Dent, J. and Kahrilas, P.J. (2004). Gastro-oesophageal reflux monitoring: review and consensus report on detection and definition of acid, non-acid, and gas reflux. Gut. 53: 1024-1031.

Tamhankar, A.P., Peters, J.H., Portale, G., Hsieh, C.C., Hagen, J.A., Bremner, C.G. and DeMeester, T.R. (2004). Omeprazole dose not reduce gastroesophageal reflux: new insights using multichannel intraluminal impedance technology. J. Gastrointest. Surg. 8: 888-896.

Thoua, N.M., Khoo, D., Kalantzis, C. and Emmanucl, A.V. (2008). Acid-related oesophageal sensitivity, not dysmotility, differentiates subgroups of patients with non-erosive reflux disease. Aliment. Pharmacol. Ther. 27: 396-403.

Vela, M.F., Camacho-Lobato, L., Srinivasan, R., Tutuian, R., Katz, P.O. and Castell, D.O. (2001). Simultaneous intraesophageal impedance and $\mathrm{pH}$ measurement of acid and nonacid gastroesophageal rflux:effect of omeprazole. Gastroenterology. 120: 1599-1606.

Zerbib, F., Duriez, A., Roman S., Capdepont, M. and Mion, F. (2008). Determinants of gastro-oesophageal reflux perception in patients with persistent symptoms despite proton pump inhibitors. Gut. 57: 156160. 